\title{
Skin and nasal vestibule colonisation by Staphylococcus aureus and its susceptibility to drugs in atopic dermatitis patients
}

\author{
Joanna Wróbel 1,B-D, Hanna Tomczak ${ }^{2,3 \mathrm{~B}-\mathrm{C}}$, Dorota Jenerowicz ${ }^{1, \mathrm{~A}, \mathrm{E}-\mathrm{F}}$, Magdalena Czarnecka-Operacz ${ }^{1, \mathrm{~A}, \mathrm{E}-\mathrm{F}}$ \\ ${ }^{1}$ Department of Dermatology, University of Medical Sciences, Poznan, Poland \\ ${ }^{2}$ Department of Genetics and Pharmaceutical Microbiology, University of Medical Sciences, Poznan, Poland \\ ${ }^{3}$ Central Laboratory of Microbiology, Heliodor Swiecicki University Hospital/University of Medical Sciences, Poznan, Poland \\ A - Research concept and design, B - Collection and/or assembly of data, C - Data analysis and interpretation, \\ $D$ - Writing the article, E - Critical revision of the article, F - Final approval of article
}

\begin{abstract}
Wróbel J, Tomczak H, Jenerowicz D, Czarnecka-Operacz M. Skin and nasal vestibule colonisation by Staphylococcus aureus and its
\end{abstract} susceptibility to drugs in atopic dermatitis patients. Ann Agric Environ Med. 2018; 25(2): 334-337. doi: 10.26444/aaem/85589

\begin{abstract}
Ibstract
Introduction. Atopic dermatitis (AD) is a chronic and recurrent disease with an inflammatory background. Genetic and environmental factors are responsible for the occurrence and development of this dermatosis.

Objective. The aim of the study was to assess the frequency of colonisation of the nasal vestibule and apparently healthy skin by S. aureus, and to assess dermal lesions for superinfection with S. aureus in AD patients.

Materials and method. The research was performed on a population of 100 AD patients. Three smears were collected for microbiological investigations: from the anterior nares, from apparently healthy skin and from lesioned skin. On collection, the material was cultured on mediums provided by the bioMerieux and Argenta companies. The strains were identified and their sensitivity to antibiotics assessed by means of the Vitek2 system (bioMerieux).

Results. S. aureus was present in at least one location in $90 \% .44 \%$ presented S. aureus colonization of apparently healthy skin and within skin lesions. In the case of $23 \%$, this particular pathogen was detected only in skin lesions, whereas $6 \%$ had a positive result within the area of apparently healthy skin. In total, S. aureus was detected in the skin of $73 \%$. In $61 \%$, apparently healthy skin had been colonized with S. aureus, whereas in $81 \%$ colonization was proved within the skin lesions. $\mathrm{S}$. aureus was detected in the nasal vestibule of $85 \%$. Among the group with S. aureus in their anterior nares, the pathogen was also found on the skin surface of $77 \%$.

Conclusions. S. aureus colonises skin in most AD patients. Most AD patients carry S. aureus in their nasal vestibules. S. aureus does not seem to colonise healthy skin as often as skin lesions in young people. The vast majority of S. aureus strains were sensitive to local antibiotics. Most $\mathrm{S}$. aureus strains produced penicillinase.
\end{abstract}

- Key words

microbiota, antibiotic resistance, atopy, skin barrier, eczema, superinfection, colonisation

\section{INTRODUCTION}

Atopic dermatitis (AD) is a chronic and recurrent disease with inflammatory background [1]. Like bronchial asthma, allergic rhinitis and conjunctivitis, it belongs to the group of atopic diseases. Genetic and environmental factors are responsible for the occurrence and development of this dermatosis [2]. There are elaborate descriptions of disorders in the structure and function of the epidermal barrier [3]. The significance of microbiological factors in the pathogenesis of eczema and the resulting therapeutic implications have been under discussion for over 100 years [4]. It is widely known that inflammation in the course of $\mathrm{AD}$ is stimulated by the antigens of skin-colonising microorganisms. Damage to the epidermal barrier facilitates their penetration into the epidermis and dermis [5]. Staphylococcus aureus (S. aureus) has a predominant role among the pathogens responsible for exacerbation of the dermatological condition in AD patients $[6,7]$.

Address for correspondence: Joanna Wróbel, Department of Dermatology, Poznan University of Medical Sciences, Poznan, Poland, Brzask 10D/112, 60-369 Poznań, Poland

e-mail: joasiaurbanska@interia.pl

Received: 11.12.2017; accepted: 21.02.2018; first published: 22.03.2018

\section{OBJECTIVE}

The aim of the study was to assess the frequency of colonisation of the nasal vestibule and apparently healthy skin by $S$. aureus, and to assess dermal lesions for superinfection with $S$. aureus in $\mathrm{AD}$ patients.

\section{MATERIALS AND METHOD}

The research was performed on a population of $100 \mathrm{AD}$ patients (48 males and 52 females) aged 2-76 years, consecutively admitted to our Department. The recruitment of patients followed the AD diagnostic criteria developed by Hanifin \& Rajka [8]. Children were qualified for the research with their parents' written consent. Three smears were collected for microbial tests: one from the nasal anterior nares, one from the skin where lesions appeared at the moment of investigation, and one from the skin which was free from eczema. Healthy skin smears were not collected from patients suffering from erythroderma.

Other patients, who had been consulted at the dermatological outpatient clinic for a follow-up examination, had no inflammatory lesions after remission of the disease. 
Patients with a history of a systemic or local antibiotic therapy in the last four weeks were excluded from the study group. Only those patients who had received the last dose at least two weeks earlier were included to the study population. Patients taking immunosupression drugs or with other kind of immunosupression were also excluded. The patients suffering from upper respiratory tract infections did not have their smear collected from the nasal vestibule to prevent false research results due to the possible predominance of another aetiological factor of the infection. Smears were collected from the following three places: the anterior nares and/or apparently healthy skin and/or diseased skin to sterile swabs which had been moistened with sterile saline for better adsorption of microorganisms from the surface of the mucosa and/or skin.

On collection, the material was cultured on solid and broth mediums provided by the bioMerieux and Argenta companies. After incubation, each medium was thoroughly analysed for the presence of $S$. aureus. The strains were identified and their sensitivity to antibiotics was assessed by means of the Vitek 2 system (bioMerieux).

\section{RESULTS}

100 of evaluated AD patients had their nasal vestibules examined for the presence of S. aureus, and their apparently healthy skin as well as skin lesions were examined. 12 patients $(12 \%)$ had only their nasal vestibules examined. 18 patients (18\%) had only their skin examined and their anterior nares were not assessed for the presence of the pathogen, 14 patients (14\%) had both their apparently healthy skin and skin lesions examined, whereas 4 patients (4\%) had only their skin lesions examined). $5 \mathrm{AD}$ patients (5\%) were examined for the presence of $S$. aureus and had their apparently healthy skin examined (there were no skin lesions during the examination). 2 patients (2\%) were examined for the presence of $S$. aureus and had their skin eczema examined (erythroderma).

Among the 100 patients, S. aureus was present in at least one location in 90 patients (90\%). In 10 patients (10\%) it was not found at any location. 44 patients (44\%) presented $S$. aureus colonization of apparently healthy skin and within skin lesions. In the case of 23 patients (23\%) this particular pathogen was detected only in skin lesions, whereas 6 patients (6\%) had a positive result within the area of apparently healthy skin. In total, $S$. aureus was detected in the skin of 73 patients (73\%). In 50 out of 82 patients (61\%), apparently healthy skin had been colonized with S. aureus, whereas in 67 out of 83 patients (81\%) colonization was proved within the skin lesions.

S. aureus was detected in the nasal vestibule of 70 out of $82 \mathrm{AD}$ patients (85\%) tested for the presence of the pathogen. Among the group of the 70 patients with S. aureus in their anterior nares, the pathogen was also found on the skin surface of 54 patients $(77.1 \%)$. Results were negative in case of the skin of 6 patients (8.6\%). 10 patients did not have their skin examined for the presence of $S$. aureus (14.3\%).

The methicillin-resistant Staphylococcus aureus (MRSA) was detected in 4 patients (4\%). 3 patients (3\%) presented colonization of their anterior nares. In the case of 1 person (1\%), colonization of apparently healthy skin was also proved. The skin surface of 2 patients (2\%) was not examined in terms of S. aureus colonization. 1 patient (1\%) presented the MRSA strain both in their apparently healthy skin and within skin lesions, whereas methicillin-sensitive Staphylococcus aureus (MSSA) was found in this patient's nasal vestibule.

Antibiograms were made for all the $S$. aureus strains cultured from the skin lesions. $90 \%$ of the strains produced penicillinase (Fig. 1). 90\% of the strains were sensitive to tetracyclines and $100 \%$ were sensitive to levofloxacin, vancomycin, teicoplanin, tigecycline and linezolid. The strains were also assessed for sensitivity to macrolides (sensitivity to erythromycin amounted to $87 \%$ ), clindamycin (91\%) and trimethoprim/sulfamethoxazole (98\%). As far as the external use drugs are concerned, the strains were sensitive to gentamicin (94\%), fusidic acid (99\%), fosfomycin and mupirocin (100\%) (Fig. 2).

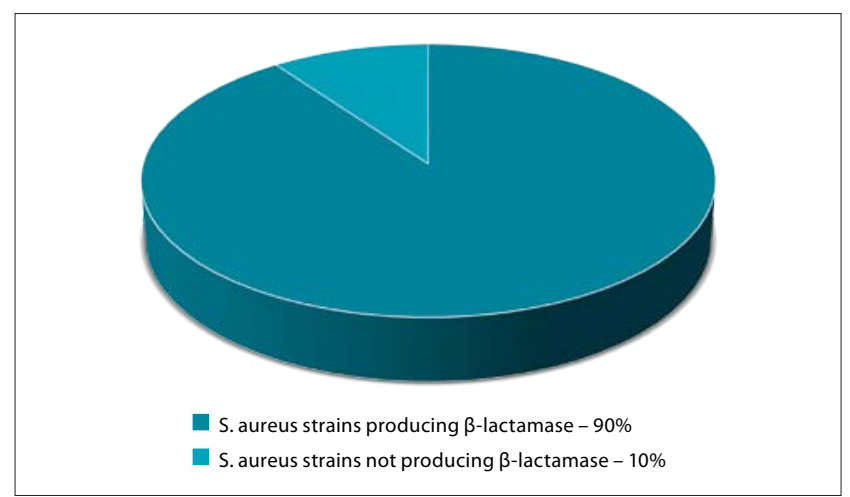

Figure 1. Antimicrobial susceptibility of Staphylococcus aureus

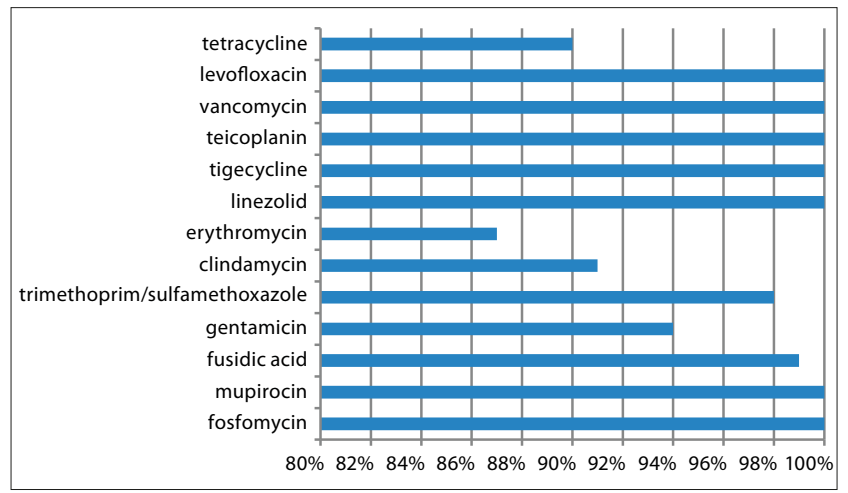

Figure 2. Sensitivity of Staphylococcus aureus to various antibiotics

As far as the nasal vestibule smear is concerned, S. aureus was more often isolated in the group of children aged under 15 years (72\%). As far as the colonisation of apparently healthy skin and the superinfection of skin lesions with S. aureus are concerned, there were higher values in the group of adults, i.e. patients aged over 15 years (54\% and 66\%, respectively). Apart from that, in the population of young people aged under 15 years the percentage of positive results of the microbial test for the presence of $S$. aureus in apparently healthy skin was twice as small than the percentage of positive results showing the presence of $S$. aureus in AD skin lesions. These values were similar in the group of patients aged over 15 years (Tab. 1). 
Table 1. Skin and nasal colonisation by Staphylococcus aureus in different age groups of patients with atopic dermatitis

\begin{tabular}{lccc}
\hline \multirow{2}{*}{ Age category (years) } & \multicolumn{3}{c}{ S. aureus colonisation in patients, $\mathrm{n}(\%)$} \\
\cline { 2 - 4 } & Nasal & Non-lesioned skin & Lesioned skin \\
\hline$<15(\mathrm{n}=32)$ & $23(72)$ & $9(28)$ & $20(62.5)$ \\
\hline$>15(\mathrm{n}=68)$ & $44(65)$ & $37(54)$ & $45(66)$ \\
\hline
\end{tabular}

\section{DISCUSSION}

The human skin is a complex ecosystem. In a surface area of about $2 \mathrm{~m}^{2}$ there is a unique diversity of habitats characterised by different physicochemical conditions, i.e. moisture, $\mathrm{pH}$ [9]. Numerous species of bacteria, fungi, mites and viruses find favourable conditions for their existence, and simultaneously make a specific microbiome [10]. The composition of this group is an individual trait [2]. It is necessary to stress the fact that most of these microorganisms are not pathogenic, but constitute a physiological protective barrier, which is important for the maintenance of skin homeostasis [11].

Skin dryness is the leading symptom of $\mathrm{AD}$ and results from the damaged epidermal barrier which causes increased transepidermal water loss. The epidermal barrier dysfunction facilitates contact between environmental allergens and cells of the skin immune system. It intensifies inflammation [3]. Moreover, barrier disruption is associated with the deficit of lipids, $\mathrm{pH}$ alkalinisation, increased serine protease activity, and decreased production of antimicrobial peptides (AMPs) [12]. All these factors favour $S$. aureus colonisation, which intensifies changes in the course of AD. There are agedependent predilection areas for the occurrence of typical AD eczemas; for example, in children these are flexion areas of knee joints, elbow, wrist and ankle joints, and the cervical fold. To a certain extent, this fact could be attributed to differences in the microbiome [9].

Latest studies prove that staphylococci, especially S. aureus, are predominant in the skin lesions of $\mathrm{AD}$ patients. The microbial diversity in skin lesions is smaller than in the skin without eczema [13]. According to the present state knowledge, S. aureus colonisation reaches $75-100 \%$ in lesional skin of $\mathrm{AD}$ patients, and $30-100 \%$ of $\mathrm{AD}$ patients have this bacteria in their nonlesional skin. By contrast, only $5-30 \%$ of healthy people are carriers of $S$. aureus $[5,14,15,16,17]$. The results of this study were consistent with thethe above observations - $S$. aureus was isolated from the skin lesions of $81 \%$ of $\mathrm{AD}$ patients.

$S$. aureus proliferates in the nasal vestibule and the skin surface may be temporarily colonised $[18,19]$. Usually, the pathogen is transferred from there to the skin, especially damaged skin, or other parts of the mucosa [19]. Colonisation with $S$. aureus is not found in all patients. This may because of the fact that about $60 \%$ of patients are temporarily colonised, and $S$. aureus may be absent at the moment of examination $[19,20]$.

If a patient carries $S$. aureus, there is higher frequency of infections with this aetiology. By contrast, the eradication of the pathogen from the nasal vestibule may decrease the incidence of infections [18]. In the presented study, $85 \%$ of AD patients (i.e. 70 patients) carried $S$. aureus in their nasal vestibules. $77 \%$ of the patients in this group, i.e. 54 patients, also carried the pathogen in their skin.

Bilal et al. studied a population of AD children and found that the colonisation of skin lesions was much more intense than the colonisation of apparently healthy skin - 38\% vs. $15 \%$ [21]. The current observations were similar. In the group of patients aged under 15 years, S. aureus was found in $62.5 \%$ of skin lesions, whereas it was found in $28 \%$ of the cases of skin without eczema. In the group of patients aged over 15 years, these values were more similar. However, it is noteworthy that the percentage of colonisation of apparently healthy skin was twice as high (54\%). Bilal et al. suggest that the presence of the bacteria on apparently healthy skin may have been caused by contamination with $S$. aureus from the anterior nares and skin lesions [21]. Hon et al. prove that the notable increase in the count of $S$. aureus obtained from nasal vestibule smears may be related with the higher total score in the SCORAD scale (Scoring Atopic Dermatitis), more extensive lesions, oozing and the presence of scabs [6]. Thus, they confirm the widely accepted thesis that $S$. aureus is the most significant pathogen in moderate to severe $\mathrm{AD}$, and the nasal cavity may be the habitat of the pathogen $[6,17,19]$. In view of this fact, it can be supposed that carrying $S$. aureus is usually the first stage of development of infection. S. aureus is undoubtedly the most common factor of bacterial infections in AD patients [6]. Hauser et al. reported that the count of $S$. aureus in the apparently healthy skin of AD patients was much greater than in skin of healthy people [22]. In the presented study, the presence of $S$. aureus in the apparently healthy skin was found in $61 \%$ of the cases. Abeck et al. also stressed the big contrast between the frequency of skin colonisation by $S$. aureus in healthy people (2-25\%) and the occurrence of these bacteria in $\mathrm{AD}$ patients, where it ranged from $76 \%$ on apparently healthy skin to as much as $100 \%$ in oozing lesions. Apart from that, they observed that colonisation of the nasal anterior nares was much more common (71\%) than colonisation of the armpits (45\%). At the same time, the study showed the significance of the nasal vestibule as the preferable habitat of $S$. aureus. In view of this fact, the researchers suggest taking anti-staphylococcal treatment into consideration. They studied the role of proteins responsible for adhesion to keratinocytes and proved that there was a wide range of proteins, such as staphylococcal protein A, coagulase, the aggregation factor and fibronectin-binding protein. The inflammation development mechanism was another aspect under discussion. Staphylococcal proteins and toxins are listed here, especially those which are superantigens [4]. The study by Goh et al. resulted in the following findings: $S$. aureus was isolated in $69.7 \%$ of eczematous lesions and $42 \%$ of cases of apparently healthy skin in AD patients. Only in $5 \%$ of the cases S. aureus was found in the control population of healthy subjects. The incidence of nasal colonisation in $\mathrm{AD}$ patients was higher than in healthy subjects, i.e. $51.5 \%$ vs $35.0 \%$. The researchers think that in view of these findings, the decision to apply an antibiotic therapy in cases of severe $\mathrm{AD}$ enables control of the course of the disease and inflammation. Systemic rather than local antibiotic therapy is recommended due to the risk of allergic contact dermatitis [23]. Jung et al. noted that most $S$. aureus strains were sensitive to a wide range of antibiotics. They proved that $S$. aureus was usually resistant to benzylpenicillin, erythromycin, clindamycin, and fusidic acid. All S. aureus strains isolated from acute lesions were sensitive to treatment with vancomycin, tigecycline, teicoplanin, linezolid and trimethoprim/sulfamethoxazole [24]. In the presented study, antibiograms revealed the lowest percentage of sensitive 
strains after the following antibiotics: erythromycin (87\%), tetracycline (90\%), $\beta$-lactam antibiotics (90\%), clindamycin (91\%), and trimethoprim/sulfamethoxazole (98\%). As far as local antibiotics are concerned, antibiograms revealed the lowest percentage of sensitive strains to gentamicin (94\%) and fusidic acid (99\%). Jung et al. compared resistance to drugs in individual age groups and noted that there was a higher percentage of methicillin-resistant strains among infants, regardless of the fact whether pathogens were isolated from acute or chronic lesions. In the group of adults, the strains were characterised by lesser sensitivity to fusidic acid, regardless of the clinical state. As far as mupirocin is concerned, the sensitivity of $S$. aureus was higher in the group of infants and children with acute lesions [24].

Researchers stress the fact that in recent years there has been increasing resistance to local antibiotics, which is undoubtedly the consequence of their abuse [25]. In the presented population of patients, the cases of carrying or being infected with the MRSA strain were predominantly found in young people (3 patients aged 16-29 years, and only one patient aged 62 years). Only one patient, i.e. a 4 -year-old boy (1\%), did not exhibit sensitivity to fusidic acid. This fact still proves very high sensitivity to the drug in comparison with the findings of the study by Jung et al. Likewise, Salah et al. proved that penicillinase-producing $S$. aureus strains were mostly isolated in $\mathrm{AD}$ patients. In the current study, these strains amounted to $90 \%$. It is noteworthy that in comparison with the global data there was a low percentage of MRSA strains among a group of Swedish patients [26]. There were 100 patients in the presented group and the presence of MRSA was confirmed in 4 patients (4\%). Adamek-Guzik et al. stress the fact that some patients should receive local antibiotic therapy to eradicate $S$. aureus from the skin and to improve the patient's clinical state [15]. Sabtu et al. suggest that during local antibiotic therapy $S$. aureus carriers and $\mathrm{AD}$ patients with infections of staphylococcal aetiology should adhere to the dosage regimen to minimise the risk of drug resistance [25].

\section{CONCLUSIONS}

1. S. aureus colonises skin in most AD patients.

2. S. aureus also colonises apparently healthy skin in AD patients.

3. Most AD patients carry $S$. aureus in their nasal vestibules.

4. S. aureus does not seem to colonise apparently healthy skin as often as skin lesions in young people (aged under 15 years).

5. The vast majority of $S$. aureus strains were sensitive to local antibiotics, i.e. fusidic acid and mupirocin.

6. Due to the fact that most $S$. aureus strains produced penicillinase (90\%) in AD patients, the application of antibiotic therapy with $\beta$-lactamase inhibitors is recommended.

\section{REFERENCES}

1. Braun-Falco O, Burgdorf WHC, Plewig G, Wolff HH, Landthaler M. [Dermatology]. 2nd ed. Lublin: Czelej; 2010. p. 426-7 (in Polish).

2. Williams MR, Gallo RL. The role of the skin microbiome in atopic dermatitis. Curr Allergy Asthma Rep. 2015; 15(11): 65, https:/doi. org/10.1007/s11882-015-0567-4.

3. Silny W. [Atopic dermatitis]. 1st ed. Poznań: Termedia; 2013. p. 54-61 (in Polish).
4. Abeck D, Mempel M. Staphylococcus aureus colonization in atopic dermatitis and its therapeutic implications. Br J Dermatol. 1998; 139: 13-16.

5. Lin YT, Wang CT, Chiang BL. Role of bacterial pathogens in atopic dermatitis. Clin Rev Allergy Immunol. 2007; 33(3): 167-77, https://doi. org/10.1007/s12016-007-0044-5.

6. Hon KL, Lam MC, Leung TF, Kam WY, Li MC, Ip M, et al. Clinical features associated with nasal Staphylococcus aureus colonisation in Chinese children with moderate-to-severe atopic dermatitis. Ann Acad Med Singapore. 2005; 34(10): 602-5.

7. Lomholt H, Andersen KE, Kilian M. Staphylococcus aureus clonal dynamics and virulence factors in children with atopic dermatitis. J Invest Dermatol. 2005; 125(5): 977-82 https://doi.org/10.1111/j.0022202X.2005.23916.x.

8. Hanifin JM, Rajka G. Diagnostic features of atopic dermatitis. Acta Dermatol Venereol. 1980; 92: 44-7.

9. Salava A, Lauerma A. Role of the skin microbiome in atopic dermatitis. Clin Transl Allergy. 2014; 4: 33, https://doi.org/10.1186/2045-7022-4-33.

10. Grice EA, Segre JA. The skin microbiome. Nat Rev Microbiol. 2011; 9(4): 244-53, https://doi.org/10.1038/nrmicro2537.

11. Zeeuwen PL, Kleerebezem M, Timmerman HM, Schalkwijk J. Microbiome and skin diseases. Curr Opin Allergy Clin Immunol. 2013; 13(5): 514-20 https://doi.org/10.1097/ACI.0b013e328364ebeb.

12. Baker BS. The role of microorganisms in atopic dermatitis. Clin Exp Immunol. 2006; 144(1): 1-9 https://doi.org/10.1111/j.13652249.2005.02980.x.

13. Seite S, Bieber T. Barrier function and microbiotic dysbiosis in atopic dermatitis. Clin Cosmet Investig Dermatol. 2015; 8: 479-83 https://doi. org/10.2147/CCID.S91521.

14. Breuer K, Haussler S, Kapp A, Werfel T. Staphylococcus aureus: colonizing features and influence of an antibacterial treatment in adults with atopic dermatitis. Br J Dermatol. 2002; 147(1): 55-61.

15. Adamek-Guzik T, Guzik TJ, Czerniawska-Mysik G, Kasprowicz A, Pryjma J. [Effects of combined therapy with oral antihistamines and topical corticosteroids on Staphylococcus aureus colonization in atopic dermatitis]. Alerg Astma Immunologia. 2002; 7(1): 33-43 (in Polish).

16. Higaki S, Morohashi M, Yamagishi T, Hasegawa Y. Comparative study of staphylococci from the skin of atopic dermatitis patients and from healthy subjects. Int J Dermatol. 1999; 38(4): 265-69.

17. Gong JQ, Lin L, Lin T, Hao F, Zeng FQ, Bi ZG, et al. Skin colonization by Staphylococccus aureus in patient with eczema and atopic dermatitis and relevant combined topical therapy: a double-blind multicentre randomized controlled trial. Br J Dermatol. 2006; 155(4): 680-7 https:// doi.org/10.1111/j.1365-2133.2006.07410.x.

18. Smith CH, Goldman RD. Staphylococcus aureus decolonization for recurrent skin and soft tissue infections in children. Can Fam Physician. 2012; 58(12): 1350-2 https://www.ncbi.nlm.nih.gov/pmc/ articles/PMC3520660/.

19. Nada HA, Gomaa NI, Elakhras A, Wasfy R, Baker RA. Skin colonization by superantigen-producing Staphylococcus aureus in Egyptian patients with atopic dermatitis and its relation to disease severity and serum interleukin-4 level. Int J Infect Dis. 2012; 16(1): 29-33, https://doi. org/10.1016/j.ijid.2011.09.014.

20. Zollner TM, Wichelhaus TA, Hartung A, Von Mallinckrodt C, Wagner TO, Brade V et al. Colonization with superantigen-producing Staphylococcus aureus is associated with increased severity of atopic dermatitis. Clin Exp Allergy. 2000; 30(7): 994-1000.

21. Bilal JA, Ahmad MI, Robaee AA, Alzolibani AA, Shobaili HA, AlKhowailed MS. Pattern of bacterial colonization of atopic dermatitis in saudi children. J Clin Diagn Res. 2013; 7(9): 1968-70, https://doi. org/10.7860/JCDR/2013/5506.3371.

22. Hauser C, Wuethrich B, Matter L, Wilhelm JA, Sonnabend W, Schopfer K. Staphylococcus aureus skin colonization in atopic dermatitis patients. Dermatologica. 1985; 170(1): 35-9.

23. Goh CL, Wong JS, Giam YC. Skin colonization of Staphylococcus aureus in atopic dermatitis patients seen at the National Skin Centre, Singapore. Int J Dermatol. 1997; 36(9): 653-7.

24. Jung MY, Chung JY, Lee HY, Park J, Lee DY, Yang JM. Antibiotic susceptibility of Staphylococcus aureus in atopic dermatitis: current prevalence of methicillin-resistant Staphylococcus aureus in Korea and treatment strategies. Ann Dermatol. 2015; 27(4): 398-403 https://doi. org/10.5021/ad.2015.27.4.398.

25. Sabtu N, Enoch DA, Brown NM. Antibiotic resistance: what, why, where, when and how? Br Med Bull. 2015; 116: 105-13, https://doi. org/10.1093/bmb/ldv041.

26. Salah LA, Faergemann J. A retrospective analysis of skin bacterial colonisation, susceptibility and resistance in atopic dermatitis and impetigo patients. Acta Derm Venereol. 2015; 95(5): 532-5 https://doi. org/10.2340/00015555-1996. 